\title{
Femtosecond Laser Surgery in Ophthalmology (2017) by Dick et al. 262 pp., 310 illustrations, Hardback, $€ 154.99 / \$ 159.99$ ISBN: 9781626232365 Thieme Publishers New York/Stuttgart
}

\author{
Randolf A. Widder ${ }^{1}$ (D)
}

Received: 30 July 2018 / Accepted: 13 August 2018 / Published online: 27 August 2018

(C) Springer-Verlag GmbH Germany, part of Springer Nature 2018

In recent years, Femtosecond laser surgery has become an innovative and important part of ophthalmic surgery. The new book "Femtosecond Laser Surgery in Ophthalmology" aims to give an extensive overview of "Femtosecond Laser Surgery in Ophthalmology". It is possible to divide the book into two parts: surgery of the cornea and surgery of the lens. The book has 29 chapters. The chapters are written by experts in their field and pioneers in femtosecond laser surgery. In addition, seven videos are provided online with a registration code. All videos come from the editors of the book and they show smile treatment, basic steps of LASIK, cataract surgery in brunescent cataract, posterior capsulotomy, surgery in paediatrics, and laser surgery with small pupils. The videos can also be found on YouTube in a higher definition.

The book describes at the beginning the basics of femtosecond technology and the beginnings of femtosecond laser in medicine and ophthalmology. After that, the first half of the book describes femtosecond surgery involving the cornea and the second half describes the newer applications in the field of cataract surgery.

Femtosecond laser-assisted in situ keratomileusis (LASIK) is described over six chapters. The author starts with a general description, moves on to clinical outcomes, complications and their management, highlights pearls in LASIK surgery, and then tries to give an outlook to the future of LASIK.

The following chapter of the book focuses on femtolaserassisted keratoplasty. This leads from penetrating surgery with different cut profiles to lamellar anterior and posterior keratoplasty.

Randolf A. Widder

r.widder@martinus-duesseldorf.de

1 Augenklinik, St. Martinus-Krankenhaus, Gladbacher Str. 26, 40219 Düsseldorf, Germany
Two chapters describe the femtosecond laser treatment of presbyopia in the cornea and in the lens.

The final chapter discusses the wide field of cataract surgery with the femtosecond laser.

The basics, the setting and the necessary infrastructure for this technique are well described so that a surgeon interested in starting cataract surgery with femtosecond laser will get a good overview.

This is followed by a thorough guidance through the crucial steps of femtosecond laser surgery in three chapters. It starts with capsulotomy and leads to lens fragmentation and corneal incisions. For the advanced user, relevant topics are discussed, for example the evolving technique of bag-in-thelens implantation or the characteristics of paediatric cataract surgery. The advanced user will also be attracted by the peculiarities of surgery in patients with ocular comorbidities. A variety of unwanted and difficult situations is discussed and "don'ts and dos" are provided.

An interesting point is the discussion about avoiding negative dysphotopsias by new lens designs which offer a groove for the anterior rhexis.

The book also offers insights into future developments of femtosecond laser surgery referring to glaucoma surgery and posterior segment surgery where the authors suspect a tremendous potential.

Last but not least, laser-induced complications and their management deserve their own chapter with valuable insight also for the advanced surgeon.

All these chapters are accompanied by a lot of helpful pictures and graphics illustrating the topics.

This book is suitable not only for beginners but also for the advanced surgeon, who can benefit from the experience of pioneers of femtosecond laser surgery.

The book might be a helpful companion in corneal and cataract surgery. 